\title{
Apoyo y Responsabilidad Medioambiental en la Comunidad de Quinrapa en el Distrito de Huanta, Perú.
}

\section{Support and Environmental Responsibility in the Community of Quinrapa in the District of Huanta, Peru.}

\author{
Maricela Manrique-Cóndor \\ Universidad Nacional Autónoma de Huanta, Perú \\ E-mail: mmanriquecondor@gmail.com ORCID: https://orcid.org/0000-0003-1888-6535 \\ Ana Fiorela Rico-Taguada \\ Universidad Nacional Autónoma de Huanta, Perú \\ E-mail: taguadafiorela123@gmail.com ORCID: https://orcid.org/0000-0002-5653-0380 \\ Mariano Anthony Cardenas-Quispe \\ Universidad Nacional de Cañete, Perú \\ E-mail: anthonycardenas02017@gmail.com ORCID: https://orcid.org/0000-0003-3033-7846 \\ Segundo Víctor Tello-Tello \\ Universidad Nacional del Callao, Perú \\ E-mail: cpcctellos@gmail.com ORCID: https://orcid.org/0000-0001-8995-1950
}

Recepción: 15/06/2021 Aceptación: 30/06/2021 Publicación: 30/07/2021

\section{Resumen}

La autoridad Nacional del Agua (ANA) es el ente encargado de gestionar sosteniblemente y multisectorial el recurso del agua. Por lo cual se busca determinar el grado el apoyo y responsabilidad medioambiental de la Autoridad Nacional del Agua (ANA) en el Distrito de Huanta, comunidad de Quinrapa. La investigación tuvo enfoque cualitativo de tipo descriptivo y diseño no experimental a nivel correlacional causal de corte transversal. La muestra estuvo conformada por 18 integrantes del comité de regantes Quinrapa, siendo el muestreo no probabilístico intencional, habiéndose excluido a los miembros de comunidades aledañas; La técnica utilizada fue la encuesta y los instrumentos utilizados fueron cuestionarios. Los resultados mostraron que el $94.4 \%$ a recibido apoyo de las autoridades, pero solo el $50 \%$ conoce sobre la gestión del ANA, En conclusión, se demostró que el Distrito de Huanta, comunidad de Quinrapa tiene apoyo de las autoridades, sin embargo, existe un desconocimiento parcial sobre la responsabilidad y gestión de la Autoridad Nacional del Agua en la zona.

\section{Palabras clave:}

Derecho humano al agua, Aprovechamiento del agua, ANA, Comunidad. 


\begin{abstract}
The National Water Authority (ANA) is the entity in charge of managing the water resource in a sustainable and multisectoral manner. Therefore, it seeks to determine the degree of support and environmental responsibility of the National Water Authority (ANA) in the District of Huanta, community of Quinrapa. The research had a qualitative descriptive approach and a non-experimental design at a cross-sectional causal correlational level. The sample consisted of 18 members of the Quinrapa irrigating committee, the sampling being non-probabilistic intentional, having excluded members of neighboring communities; The technique used was the survey and the instruments used were questionnaires. The results showed that $94.4 \%$ have received support from the authorities, but only 50\% know about the management of the ANA In conclusion, it was shown that the District of Huanta, Quinrapa community has support from the authorities, however there is a lack of knowledge partial on the responsibility and management of the National Water Authority in the area.
\end{abstract}

\title{
Keywords:
}

Human right to water, Water use, ANA, Community.

\section{Resumo}

A Autoridade Nacional de Águas (ANA) é a entidade responsável pela gestão do recurso hídrico de forma sustentável e multissetorial. Assim, pretende-se determinar o grau de apoio e responsabilidade ambiental da Autoridade Nacional de Águas (ANA) no Distrito de Huanta, comunidade de Quinrapa. A pesquisa teve abordagem qualitativa descritiva e delineamento não experimental em nível de correlação causal transversal. A amostra foi composta por 18 membros do comitê de irrigação da Quinrapa, sendo a amostragem não probabilística intencional, sendo excluídos membros das comunidades vizinhas; A técnica utilizada foi a survey e os instrumentos utilizados foram questionários. Os resultados mostraram que $94,4 \%$ receberam apoio das autoridades, mas apenas $50 \%$ conhecem a gestão da ANA. Concluindo, foi demonstrado que o Distrito de Huanta, comunidade de Quinrapa tem apoio das autoridades, no entanto falta conhecimento parcial sobre a responsabilidade e gestão da Autoridade Nacional de Águas na área.

\section{Palavras chave:}

Direito humano à água, Uso da água, ANA, Comunidade.

\section{Introducción}

La Autoridad Nacional del Agua es el organismo público encargado de administrar, conservar y proteger los recursos hídricos en sus fuentes naturales, tales como, ríos, lagos, lagunas, acuíferos. Tiene como misión ejercer la rectoría técnica - normativa y 


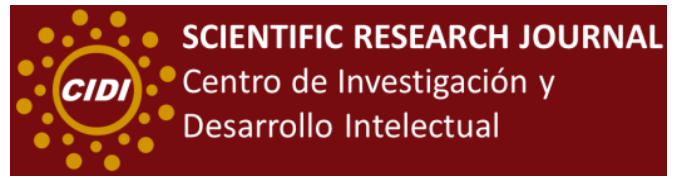

establecer procedimientos para la gestión integrada, sostenible y multisectorial de los recursos hídricos en beneficio de los usuarios de agua y población en general, de manera oportuna y eficaz (Autoridad Nacional del Agua, 2018). En la región de Ayacucho, provincia de Huanta, distrito de Huanta y comunidad de Quinrapa, La Autoridad Nacional del Agua (ANA) viene trabajando con las comunidades de los centros poblados, mediante los comités de regantes, quienes trabajan a favor de su población y preservación del agua, siendo así un buen factor de trabajo en equipo.

El agua, uno de los recursos naturales más importantes, y a la vez el más escaso y variable en nuestro país. Ello es así especialmente en la costa y en la sierra, que son las regiones donde se asentaron las principales culturas prehispánico y donde se desarrolló la agricultura de riego. Sin embargo, esta escasez y variabilidad se deben a nuestras particularidades condiciones geográficas, extremadamente diversas y accidentadas, y a nuestras fluctuantes condiciones climáticas uno de cuyos resultados son los ciclos periódicos de inundaciones y sequías. (Vilca, 2017).

El uso de aguas con fines de generación de energía eléctrica y de la conservación de los caudales ecológicos confirman la naturaleza concesional de las mencionadas licencias, por lo cual es necesario que la ANA asuma un rol protagónico en toda actividad referida al uso o gestión de los recursos hídricos. (Mendoza, 2020)

La participación de la Autoridad Nacional del Agua en la gestión de las aguas residuales urbanas tiene la función de ente rector que comprueba, registra e inspecciona el cumplimiento de las disposiciones de la calidad del agua, ya que el agua es uno de los recursos naturales sustanciales y que forma parte importante en el crecimiento demográfico de una nación; este compuesto químico a su vez es el más considerable del planeta y resulta necesario para el desarrollo de la vida humana. (Diaz, 2018)

La presente investigación plantea como problema fundamental, la siguiente pregunta general; ¿Cuál es el apoyo y Responsabilidad Medioambiental de la ANA en el Distrito de Huanta-Comunidad de Quinrapa? En este contexto, se plantea el objetivo de determinar el grado el apoyo y Responsabilidad Medioambiental de la ANA en el Distrito de Huanta-Comunidad de Quinrapa. 


\section{Revisión de la literatura}

\subsection{La Autoridad Nacional del Agua}

La autoridad nacional del agua, perteneciente al ministerio de agricultura y riego, según la ley N²9338 - Ley de Recursos Hídricos, es el ente director y máxima autoridad técnico normativa el Sistema Nacional de gestión de los Recursos Hídricos, y además forma parte del Sistema Nacional de gestión Ambiental (Autoridad Nacional del Agua, 2021b).

\subsection{Derecho Humano al Agua}

El agua es vital para la vida humana y la sustentabilidad de nuestro mundo. Esto es debido a sus diversos usos o también llamado "polifuncionalidad", ya que este recurso da la posibilidad de consumo, hábitos de limpieza e higiene, cultivo de alimentos, producir energía, procesar minerales y otras actividades de industria, hasta interviene en el transporte de otros alimentos mediante embarcaciones (Carvajal, 2018). Por lo tanto, el agua un recurso indispensable en muchos ámbitos de las actividades humanas. Como menciona (Pari-Bedoya, 2019) que el agua es un elemento esencial y base para el desarrollo de los derechos fundamentales del ser humano.

\subsection{Bienestar}

En la mayoría de las investigaciones es un concepto referenciado a la buena salud física y mental, sin embargo, este concepto pocas veces se relacionó con la satisfacción de haber alcanzado una meta importante (Casanova, 2016). Esto quiere decir que esta palabra está definida más por la buena salud humana.

\subsection{Comunidad}

(Vanessa \& Agudelo, 2017) mencionan que una comunidad es un sentido de pertenencia mediante la participación del individuo, ya que puede residir en la comunidad, pero no participar ni pertenecer a esta. Esto significaría que una persona que reside en una comunidad pero que no participa en ella, no adoptaría ningún sentido de pertenencia. 


\section{Materiales y Métodos}

La investigación tuvo enfoque cualitativo, tipo descriptivo, diseño no experimental, nivel correlacional causal, de corte transversal. la muestra conformó 18 miembros del comité de regantes de Quinrapa, entre hombres y mujeres de 35 años a más, con un promedio 30 años dedicados a esta labor, siendo el muestreo no probabilístico intencional, habiéndose excluido a los miembros regantes de comunidades aledañas; La técnica utilizada fue la encuesta y los instrumentos utilizados para cada variable fueron cuestionarios.

\subsection{Estructura de la encuesta}

Se diseñó una encuesta compuesta por 07 preguntas agrupadas en 3 secciones: 1) identificación general del regante, 2) conocimiento de la ANA ,3) análisis de la situación del agua en la comunidad de Quinrapa.

\section{Tabla 1}

Estructura de la encuesta.

\begin{tabular}{|c|c|}
\hline SECCIÓN & TIPO DE PREGUNTA \\
\hline & 1. Edad \\
\hline \multirow[t]{2}{*}{ Identificación general del regante } & 2. Género \\
\hline & 3. Tiempo que lleva como regante \\
\hline \multirow[t]{3}{*}{ Conocimiento del ANA } & 4. ¿Conoce la gestión de la ANA? \\
\hline & $\begin{array}{l}\text { 5. Apoyo de las autoridades hacia los } \\
\text { regantes. }\end{array}$ \\
\hline & $\begin{array}{l}\text { 6. Proyecto de mejora de los canales de } \\
\text { riego. }\end{array}$ \\
\hline comunidad de Quinrapa & $\begin{array}{l}\text { 7. Situación actual del agua dirigido para } \\
\text { riego en la comunidad de Quinrapa. }\end{array}$ \\
\hline
\end{tabular}

\section{Resultados}


Figura 1

Conoce la Gestión de la ANA

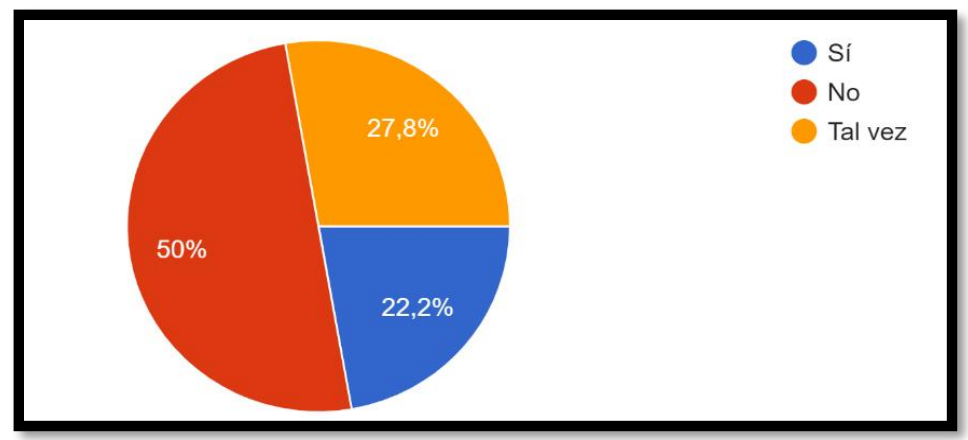

En la figura 1, nos muestra la encuesta realizada sobre el conocimiento de la gestión de la ANA, un $50 \%$ dice que no lo conoce, un $22,2 \%$ dice que si lo conoce y un $27,8 \%$ dice que tal vez lo conoce.

\section{Figura 2}

Apoyo de las Autoridades hacia los Regantes de la Comunidad de Quinrapa.

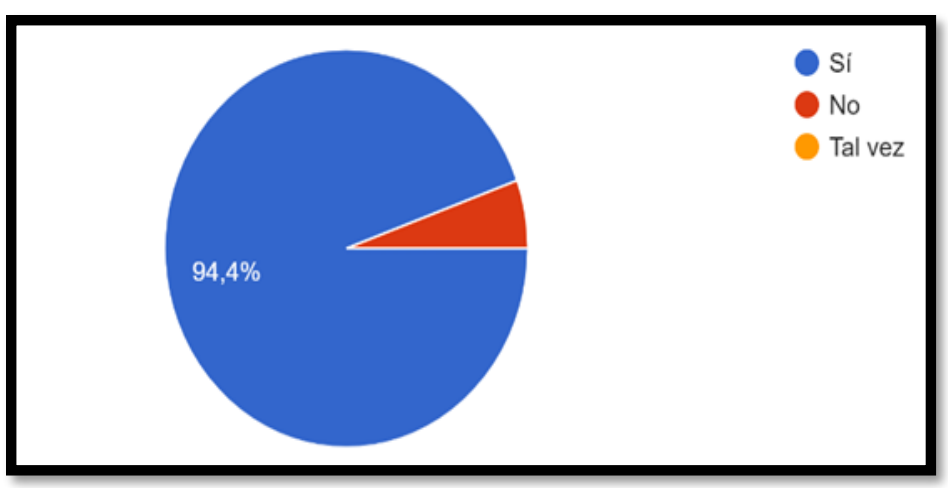

En la figura 2 se evidencia que $94.4 \%$ de la muestra encuestada mencionan que las autoridades sí les brinda apoyo y el 5.6\% dice que no.

\section{Figura 3}

Conocimiento de algún Proyecto para la mejora de los Canales de Riego. 


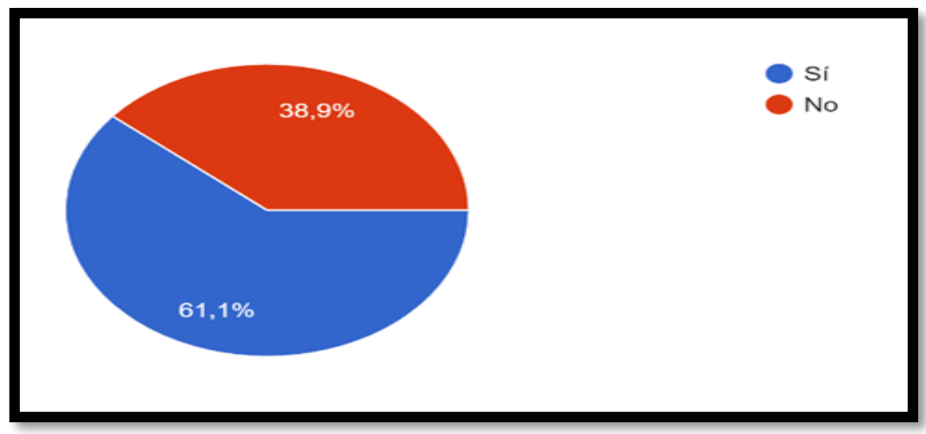

De acuerdo con la muestra encuestada, en la tabla 6 se muestra que el $61,1 \%$ de los regantes mencionan que sí y 38,9\% que no.

\section{Figura 4}

Situación actual del Agua dirigido a Riego en la Comunidad de Quinrapa.

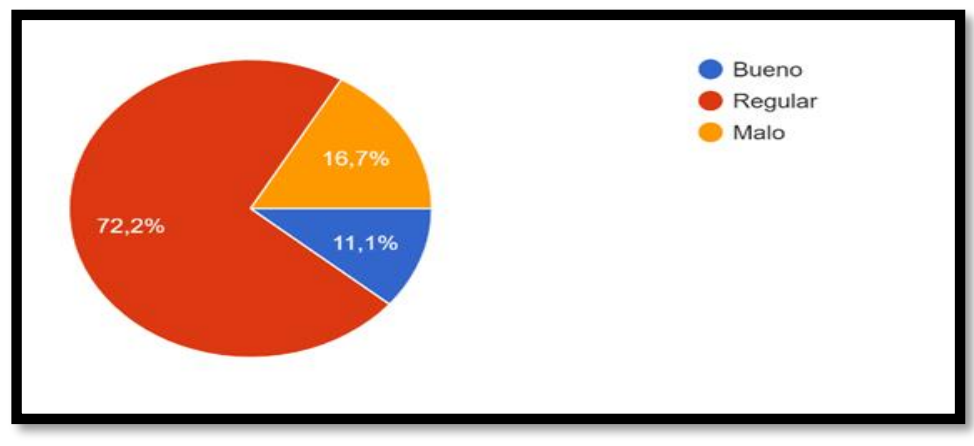

En la tabla y figura 7 se evidencia que el $11,1 \%$ de los regantes mencionan que es bueno, el $72,2 \%$ dijeron que es regular y el $16,7 \%$ dijeron que es malo.

\section{Discusión}

Según el resultado 1, el 50\% afirma conocer a la autoridad nacional del agua, esto sería causado por que la institución no estaría invirtiendo en publicidad, lo que originaria un desconocimiento de la autoridad. Como mencionan (Falquez Arce et al., 2017) que incrementar el gasto en publicidad aumenta la demanda. Lo que significaría una percepción positiva por el usuario final.

Según el resultado 2, el 94.4\% si recibe apoyo, lo que significaría que las autoridades locales si estuviesen gestionando la distribución de riego y cumpliendo con la norma. Como menciona la (Autoridad Nacional del Agua, 2021a) en la página web del gobierno 
del Perú, que una de sus actividades es autorizar tomos de agua que se utilizaran y/o distribuirán los EPS y juntas de regantes.

Según el resultado 3, el 61.1\% conoce sobre alguna mejora en los canales de riego, lo que representaría de que las obras no se están anunciando correctamente. Como mencionan (Yánez Balarezo \& Cusot, 2018) que la publicidad se usa para dar a conocer la gestión, posicionamiento de la institución y logros obtenidos del gobierno, obtener acciones y/o variación de actitud de los ciudadanos en temas relacionados a la institución.

Según el resultado 4, el 72.2\% de afirma que la situación actual del agua dirigido al riego es regular, esto se debería que la gestión del agua en la zona se está realizando con una eficiencia baja. (Gil-Marín et al., 2018) mencionan que es importante realizar revisión, evaluación, investigación y manejo para asegurar una gestión de calidad.

\section{CONCLUSIONES}

De acuerdo con la investigación queda demostrado que en el Distrito de HuantaComunidad de Quinrapa, si tiene el debido apoyo por parte de las autoridades, sin embargo, se desconoce la responsabilidad Medioambiental de parte de la ANA y su gestión de proyectos en el distrito.

\section{REFERENCIAS BIBLIOGRÁFICAS}

Carvajal, M. (2018). El derecho Humano al agua: Análisis histórico, contenido y alcance en la legislación chilena [Universidad de chile]. In Universidad Nacional de Chile. http://www.chilesustentable.net/wp.content/uploads/2010/06/CONFLICTOS-porel-agua-en-chile-362-pp-en-baja1.pdf>

Casanova-Rodríguez, C. L. (2016). Estudios de Bienestar en la comunidad universitaria de la residencia estudiantil en la Universidad de Cienfuegos. Revista Universidad y Sociedad, Seriada en línea, 137-141. http://rus.ucf.edu.cu/

Diaz-Chilet, S. V. (2018). Rol fiscalizador de la Autoridad Nacional del Agua en la gestión de las aguas residuales urbanas en Puerto Huarmey en el año 2017. [Universidad Cesar Vallejo]. In Universidad Cesar Vallejo. 
Mendoza-Flores, S. E. (2020). El régimen de utilización de aguas públicas en el Perú: estudio sobre la naturaleza concesional de la licencia de uso de aguas. [Pontificia Universidad Católica del Perú]. In Pontificia Universidad Católica del Perú.

Autoridad Nacional del Agua. (2018). Servicios y Plataforma de información. [Presentación de Power Point]. Repositorio Digital de Recursos Hídricos. https://hdl.handle.net/20.500.12543/3031

Pari-Bedoya, I. N. (2019). El Derecho al Agua en el Perú desde el nuevo constitucionalismo latinoamericano. [Universidad Privada de Tacna]. In Universidad Privada de Tacna.

Vilca-Pérez, E. M. (2017). La gestión del agua de riego en la cuenca del rio Cabanillas, orientada a la política y estrategia nacional de recursos hídricos del Perú. [Universidad Nacional del Altiplano]. In Universidad Nacional del Altiplano.

Autoridad Nacional del Agua. (30 de Julio del 2021a). Autoridad Nacional del Agua - ¿Qué hacemos? / Gobierno del Perú. Gobierno Del Perú. https://www.gob.pe/4404autoridad-nacional-del-agua-que-hacemos

Autoridad Nacional del Agua. ( 30 de Julio del 2021b). Nosotros / Drupal. https://www.ana.gob.pe/nosotros/la-autoridad/nosotros

Falquez Arce, C., Silva Guerrero, B., \& Rojas Herrera, V. (2017). La inversión en publicidad y su efecto en las Medianas Empresas de Guayaquil. RETOS. Revista de Ciencias de La Administración y Economía, 7, 99-112. https://www.redalyc.org/jatsRepo/5045/504553252011/html/index.html

Gil-Marín, J. A., Vizacíno, C., \& Montaño-Mata, N. J. (2018). Evaluacion De La Calidad Del Agua Superficial. Anales Científicos UNALM, 79(1), 111-119.

Vanessa, P., \& Agudelo, S. (2017). La participación, la responsabilidad social y la vida en comunidad como ejes para abordar la intimidación escolar. Pensamiento 
Psicológico, 15(2), 121-132]. https://doi.org/10.11144/Javerianacali.PPSI15-2.prsv

Yánez-Balarezo, S., \& Cusot, G. (2018). Análisis del uso de la publicidad en las organizaciones de gobierno: información, posicionamiento y propaganda. MARCO (Márketing y Comunicación Política). https://doi.org/10.15304/marco.4.5291 